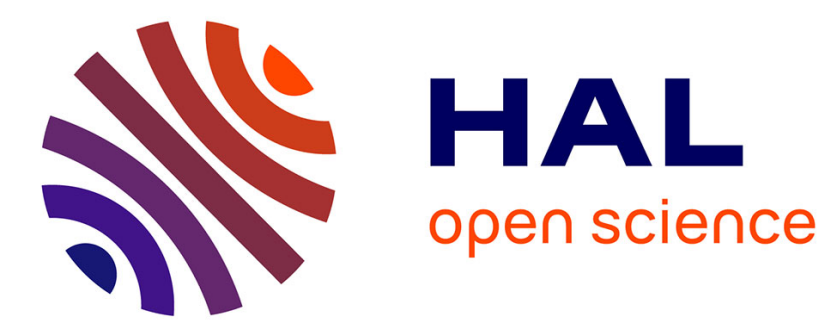

\title{
La démocratie japonaise entre crise et réinvention
}

\author{
Jean-Marie Bouissou
}

\section{To cite this version:}

Jean-Marie Bouissou. La démocratie japonaise entre crise et réinvention. Revue d'Etudes Comparatives Est-Ouest, 1997, 28 (3), pp.63-93. 10.3406/receo.1997.2867 . hal-01010331

\section{HAL Id: hal-01010331 \\ https://hal-sciencespo.archives-ouvertes.fr/hal-01010331}

Submitted on 19 Jun 2014

HAL is a multi-disciplinary open access archive for the deposit and dissemination of scientific research documents, whether they are published or not. The documents may come from teaching and research institutions in France or abroad, or from public or private research centers.
L'archive ouverte pluridisciplinaire HAL, est destinée au dépôt et à la diffusion de documents scientifiques de niveau recherche, publiés ou non, émanant des établissements d'enseignement et de recherche français ou étrangers, des laboratoires publics ou privés. 


\title{
La démocratie japonaise entre crise et réinvention
}

\author{
In: Revue d'études comparatives Est-Ouest. Volume 28, 1997, º3. pp. 63-93.
}

\section{Résumé}

La démocratie japonaise traverse une profonde crise de légitimité, qui affecte l'ensemble du système politique. Pourtant s'affirme, à partir du local, un renouveau citoyen à travers la multiplication des réseaux associatifs, centrés autour des coopératives autogestionnaires de consommation. Bien que cette évolution soit fragile du fait de leur manque de coordination au niveau national, de l'étroitesse de leur base sociologique et de l'ambiguïté de leurs relations avec les différents pouvoirs, ces formes alternatives de participation imprègnent peu à peu le discours et les pratiques politiques actuelles, qui s'articulent autour des idées de décentralisation et de proximité. Ce mouvement pourrait constituer un contrepoint à l'ultra- libéralisme ambiant et permettrait au Japon de développer une expérience originale à travers laquelle, pour la première fois de son histoire, il participerait à la recomposition de la scène idéologique mondiale.

\section{Abstract}

Japanese democracy between crisis and reinvention.

Japanese democracy is in the throes of a crisis of legitimacy that has shaken up the whole political system. At the local level however, a growing number of associations centered around self-managed consumer cooperatives are reviving the idea of citizenship. Though still frail, given the lack of coordination at the national level, their narrow social bases and their ambiguous relations with public authorities, these alternative forms of participation in public life are gradually permeating political practices and discourses, which revolve around the ideas of decentralization and proximity. This movement might serve as a counterweight to the ambient far-liberalism. Japan might thus be making an original experiment that, for the first time in its history, would give it a part in changing ideologies at the world-level.

Citer ce document / Cite this document :

Bouissou Jean-Marie. La démocratie japonaise entre crise et réinvention. In: Revue d'études comparatives Est-Ouest. Volume 28, 1997, N³. pp. 63-93.

doi : $10.3406 /$ receo.1997.2867

http://www.persee.fr/web/revues/home/prescript/article/receo_0338-0599_1997_num_28_3_2867

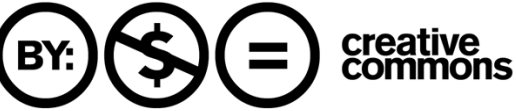




\title{
La démocratie japonaise entre crise et réinvention
}

\author{
Jean-Marie BOUISSOU *
}

\section{UNE DÉMOCRATIE EN CRISE}

\subsection{La crise de la démocratie japonaise...}

La démocratie japonaise semble aujourd'hui minée par le haut. La perte de légitimité de la représentation nationale se traduit de multiples façons.

a) La participation électorale recule très fortement depuis le début de la décennie. L'abstention aux législatives était en moyenne de $28,2 \%$ dans les années $1960,28,9 \%$ dans les années 1970 et 28,7\% dans les années 1980. En 1993, elle est passée à 32,8\% et en 1996 à 40,4\%. Aux sénatoriales, où les Japonais ont toujours moins voté, elle est passée de $35 \%$ en 1989 à $49,7 \%$ en 1992, pour atteindre un record absolu en 1995, quand plus de la moitié des électeurs $(54,5 \%)$ n'ont pas voté pour la première fois de l'après-guerre. Jamais on n'avait observé une telle ampleur ni une telle constance de la progression de l'abstention, à quatre sénatoriales et deux législatives successives. A titre de comparaison, "1'abstention réelle" en France l n'était que de $34,8 \%$ aux législatives de 1997.

b) Le vote de protestation a progressé parallèlement. Il s'est porté par priorité sur le Parti communiste japonais (PCJ), qui est passé de 7,7\% aux législatives de 1993 à $13 \%$ en 1996. Le taux des votes blancs et nuls s'est aussi accru spectaculairement. Aux législatives, il est passé de $0,5 \%$ en 1990 à $0,8 \%$ en

\footnotetext{
* Chercheur au CERI, Fondation Nationale des Sciences Politiques (27, rue SaintGuillaume, 75337 Paris Cedex 07). Cet article doit beaucoup à deux mois d'enquête menée auprès des mouvements alternatifs japonais en 1996, avec le soutien de la Fondation du Japon, que je tiens à remercier ici.

1. Compte tenu du fait qu'environ $9 \%$ du corps électoral ne s'inscrit pas. Au Japon, l'inscription est automatique.
} 
1993, puis a bondi en $1996(4,3 \% \text { et } 6 \%)^{2}$. Aux sénatoriales, il passe de 2,5\% en 1989 à 6,5\% en 1995.

c) La volatilité de l'électorat est aussi extrême. L'observation des résultats électoraux n'en donne qu'une idée très imparfaite. Les sondages menés à intervalles réguliers par certaines équipes universitaires sur des populations d'électeurs-témoins révèlent un phénomène beaucoup plus profond.

d) L'identification partisane a littéralement volé en éclats, quel que soit l'indicateur qu'on utilise pour la mesurer. Le pourcentage des électeurs qui affirment ne soutenir aucun parti est passé de $31,3 \%$ en 1990 à près de $60 \%$ à la veille des législatives de 1996. La qualité de l'attachement partisan, chez ceux qui en affichent encore un, s'est dramatiquement détériorée, et les motivations qu'ils invoquent sont désormais surtout "négatives" (soutien par défaut) ${ }^{3}$.

e) Les sondages sur la confiance accordée aux politiciens ont atteint le fond avec seulement $1 \%$ des électeurs qui font "tout à fait confiance" aux politiciens et $15 \%$ qui leur font "plutôt confiance" 4 .

f) Les élections locales de 1995 ont vu le triomphe de candidats qui ont fait campagne sur le rejet de l'establishment politico-bureaucratique et l'appel au "peuple de la communauté locale". Les deux postes majeurs de gouverneurs à Tokyo et Osaka ont été enlevés par deux vedettes du petit écran, candidats indépendants. Leur succès est d'autant plus significatif qu'il a été acquis sans le soutien d'aucun parti et contre des candidats "quasi officiels", qui venaient de la très haute administration et qui étaient soutenus par l'ensemble des partis politiques, depuis les socialistes jusqu'à la droite la plus conservatrice.

Résultat : le système des partis a volé en éclats. Sur les huit formations politiques représentées au Parlement en 1993, seulement deux subsistent aujourd'hui (le PLD - Parti libéral démocrate - et le PCJ - Parti communiste japonais). Deux autres sont réduites à l'état de fantômes (le PSDJ - Parti socialiste - a 15 députés et le PP - Parti pionnier - seulement 2). Quatre ont disparu pour laisser place à deux nouveaux partis. Mais le plus important de ceux-ci, le NPP (Nouveau parti du progrès), a commencé à se déliter dès le lendemain des élections de 19965.

2. $4,3 \%$ dans les 300 circonscriptions locales et $6 \%$ pour les 200 sièges pourvus à la proportionnelle. Étant donné la réforme du mode de scrutin en 1994, seul le premier chiffre peut être comparé à ceux des élections précédentes. Calculs de l'auteur par différence entre le nombre des votants et le nombre des suffrages recueillis par les candidats (les statistiques japonaises ne décomptent jamais les votes blancs et nuls).

3. Pour les chiffres détaillés, voir Jean-Marie Bouıssou, "Les évolutions des facteurs du choix électoral au Japon", Revue Internationale de Politique Comparée, vol. 3 (3), décembre 1996, pp. 607-627.

4. Sondage du quotidien Asahi Shinbun du 19 mars 1996.

5. Pour le détail de cette recomposition, voir Jean-Marie Bouissou, "Japon : la fin des turbulences?", Pouvoirs, n 82 , septembre 1997, pp. 177-187. 
Le Japon est aujourd'hui gouverné par un cabinet PLD homogène, mais minoritaire. Minoritaire à la Chambre, où il lui manque 12 sièges, et au Sénat, où il lui en manque 16. Encore bien plus minoritaire dans le pays, où il n'a obtenu le 20 octobre 1996 que $18,6 \%$ des voix du corps électoral - dont $40,4 \%$ s'est abstenu et $41 \%$ a voté pour un parti de l'opposition 6 .

A titre de comparaison, le gouvernement issu des élections du ler juin 1997 en France est soutenu par environ $30 \%$ du corps électoral 7.

Plus grave, cette délégitimation affecte toutes les élites. Le patronat a été largement déconsidéré par les excès spéculatifs de la période "d'économiecasino" (1985-1990) et les innombrables scandales qui ont suivi l'éclatement de la bulle spéculative, dont ceux qui secouent aujourd'hui la maison de titres Nomura et la banque Dai-Ichi Kangyô sont les plus récents, mais assurément pas les derniers. La haute administration, à laquelle ont été attribués les succès du miracle économique japonais et qui en tirait un immense prestige, est encore plus déconsidérée. Depuis 1980, une partie du patronat, inspiré par le libéralisme reagano-thatchérien, a lancé une offensive contre les pouvoirs de régulation économique de la bureaucratie, en prônant les privatisations et la "réforme administrative" (gyôsei kaikaku). Au début des années 1990, avec la crise économique et les progès d'une mondialisation qui rendait de plus en plus obsolètes le système protectionniste et anti-concurrentiel géré par les grands ministères, cette offensive a été élargie à l'exigence d'une "déréglementation" générale (kiseikanwa). Elle a trouvé un puissant écho dans l'opinion, traumatisée par l'épisode spéculatif, dont le Ministère des finances a été jugé responsable 8 . Elle traduit aussi une évolution profonde de la société, qui s'éloigne des valeurs sur lesquelles reposait la "société verticale" (tate shakai) ou "corsetée" (kanri shakai), autoritaire et hiérarchique, respectueuse d'un pouvoir dont les fonctionnaires étaient l'incarnation 9. Taxée d'incompétence et de collusion avec les intérêts privés, secouée par les scandales, attaquée en justice par les contribuables, l'administration est aujourd'hui sur la défensive. Bien que la plupart des politiciens entretiennent traditionnellement des liens étroits avec les ministères, ils doivent tous renchérir de rhétorique anti-bureau-

6. Les chiffres sont calculés d'après les suffrages exprimés à la proportionnelle. Le PLD a fait mieux pour les 300 sièges locaux $(38,6 \%$ des suffrages contre $32,8 \%$ à la proportionnelle), mais cela n'est pas significatif car il était le seul grand parti à aligner 300 candidats.

7. Compte tenu de "l'abstention réelle" et avec la méthode de calcul des voix obtenues "au tour décisif" (voir Le Monde du 5 juin 1997, p. 10).

8. Pour plus de détails, voir Jean-Marie Bousssou, "La réforme administrative et la chute du PLD", Revue d'Administration Publique, n' 73 (L'administration japonaise), janvier-mars 1995, pp. 9-24.

9. Pour l'ensemble de cette évolution, voir Jean-Marie BouIssou et Éric SEIzeler, "La société et la mutation du système de valeurs", in Jean-Marie Bouissou, François Gipouloux et Éric Seizelet, éds., Japon : le déclin?, Bruxelles : Complexe, 1996, pp. 57-100. 
cratique. En l'état actuel de l'opinion, aucun discours politique n'est plus audible s'il ne s'articule pas autour d'une attaque contre "les pouvoirs excessifs de l'administration". Sur ce sujet, la démagogie ne connaît plus de limites: l'éphémère gouvernement Hata (mai-juin 1994) avait promis que les prix de détail baisseraient de $30 \%$ grâce à la "déréglementation" et, pendant la campagne législative de 1996, le PLD a proposé de réduire le nombre des ministères de vingt à dix...

\section{2. ... variante de la crise des démocraties à partis ultra-dominants depuis la fin de la Guerre froide}

La crise de légitimité qui affecte le système politique japonais n'est pas spécifique. Elle participe de la remise en cause générale qui affecte, à des degrés divers, toutes les démocraties depuis que la fin de la Guerre froide les a privées du repoussoir communiste. Elle frappe tout particulièrement celles qui s'organisaient autour d'un parti ultra-dominant : l'Italie de la Démocratie chrétienne et le Japon du Parti libéral démocrate. Les raisons en sont faciles à comprendre.

a) Un parti ultra-dominant pratique par nature une stratégie "attrape-tout". Il est donc très vulnérable à la réactivation des conflits d'intérêts provoquée par la mondialisation de l'économie. Au Japon, ce sont ces conflits (notamment ceux qui opposent les grandes entreprises "mondialisées" et les secteurs sousproductifs et sur-protégés) qui sous-tendent les attaques contre l'administration 10. L'autre arme par excellence d'un parti ultra-dominant est la distribution massive des biens publics, dont il dispose sans contrôle. Il pâtit donc particulièrement dans le cas d'une réduction des ressources budgétaires, comme celle que peut entraîner un ralentissement de la croissance. Au Japon, le déficit budgétaire atteignait 6,75 \% du PIB en 1996. Il impose la diminution des subventions aux clientèles protégées du PLD, notamment les agriculteurs, et celle des budgets de travaux publics, qui constituent traditionnellement l'arme absolue des députés conservateurs dans leurs fiefs locaux.

b) Tout parti ultra-dominant est très vulnérable à "l'effet de surcharge". Quand les demandes sociales se diversifient et deviennent moins compatibles entre elles, un parti "attrape-tout" ne parvient plus à accommoder trop d'intérêts trop divergents. En outre, plus la société se modernise, plus le délai de réaction exigé des décideurs se réduit, à cause des communications instantanées et de la volatilité d'une opinion qui réagit instantanément aux images, faute de pouvoir "traiter" trop d'informations trop complexes. Par conséquent, le processus de décision devient de moins en moins efficace (ou du moins, il

10. Pour cette réactivation des conflits dans le cas du Japon, voir Jean-Marie Bousssou, "La recomposition politique au Japon. Entre 'rien n'a changé' et 'tout est possible", Pouvoirs, n 71 (Le Nouveau Japon), 1994, pp. 53-66. 
est perçu comme tel), jusqu'à une quasi-paralysie. Ce phénomène est très net dans le Japon des années 1980, où les commissions gouvernementales identifient clairement les chantiers des réformes à ouvrir (fiscalité, éducation, mode de scrutin, réforme administrative...) sans que le PLD soit capable d'en faire aboutir un seul.

c) Pour "ratisser" le plus large possible, les partis ultra-dominants ont été conduits à adopter un profil idéologique très bas, souvent réduit au plus petit dénominateur commun qu'était la dénonciation rituelle du "péril communiste". Ils sont donc mal préparés à satisfaire les "demandes émotionnelles" croissantes d'un électorat pour lequel les enjeux traditionnels se brouillent et qui fonctionne de plus en plus "aux images". Dans le cạs du PLD, cette incapacité est renforcée par son mode interne de gestion des rivalités d'ambitions, qui l'amène depuis un quart de siècle à porter au gouvernement des personnages falots, pour des durées limitées 11 .

d) Tout parti ultra-dominant, une fois privé de la légitimation fournie par l'anti-communisme, est particulièrement vulnérable aux sanctions d'ordre éthique. Car plus il est resté au pouvoir sans le contrôle d'une alternance, plus ses relations avec les autres élites, et avec ses clientèles électorales, ont tourné à la corruption structurelle 12. Après 1989, c'est la multiplication des scandales qui a progressivement paralysé les gouvernements PLD. Elle a fourni le prétexte à la scission qui a écarté le PLD provisoirement du pouvoir après juillet 1993 et qui a mis en route le processus d'une recomposition à grande échelle du système des partis.

e) Tout parti ultra-dominant draine, au fil des années, le plus grand nombre de ceux qui ambitionnent de faire carrière en politique. Il est donc particulièrement vulnérable aux conflits internes d'ambitions, surtout depuis que les contraintes de la Guerre froide (faire front contre le communisme) ont disparu. Dans le cas du PLD, il est facile de montrer que les scissions et les reclassements de 1993, ostensiblement justifiés au nom des principes de la morale publique, se sont en réalité effectués selon des critères uniquement relatifs au jeu des ambitions, en fonction des plans de carrière des politiciens et des ressources personnelles dont chacun disposait 13 .

11. Pour plus de détails, voir Asaharu KoHNO, "Rational Foundation for the Organization of the LPD", World Politics, 44 (3), April 1992, pp. 369-387. En français, JeanMarie BouIssou, "Les cassures du PLD. Les conflits d'ambitions et leur gestion dans le parti gouvernemental japonais", in Jean-Marie Bouissou, dir., L'envers du consensus. Les conflits et leur gestion dans le Japon contemporain, Paris : Presses de Sciences Po, 1997, pp. 37-56.

12. Jean-Marie BouIssou, "Gifts, Networks and Clienteles : Corruption in Japan as a Redistributive System", in Yves Meny and Donatella Dellaporta, dirs., Democracy and Corruption in Europe, London : Pinter, 1997, pp. 132-147.

13. Junko KATO, "When the Party Breaks Up", communication au Congrès de l'American Political Science Association, Chicago, août 1995. En français, Jean-Marie Bourssou, "Les élections législatives japonaises du 18 juillet 1993", Revue Française de Science Politique, vol. 44 (3), pp. 379-423. 


\section{RÉINVENTER L'INTERACTION CITOYENNE AU NIVEAU LOCAL : LES COOPÉRATIVES DE CONSOMMATION AUTOGESTIONNAIRES 14}

Tant par les chiffres à travers lesquels on peut l'apprécier que par son aspect structurel, la crise du système politique japonais apparaît donc profonde. Pourtant, dans ce contexte, on voit s'affirmer de nouvelles pratiques de la démocratie, qui attestent son enracinement et dont le caractère le plus significatif est de s'exercer par priorité au niveau local.

\subsection{Les communautés locales, "écoles de la démocratie"}

Grâce aux Américains, qui voulaient en faire "l'école de la démocratie", les citoyens japonais jouissent de possibilités d'action très étendues dans leurs communes et leurs 47 "départements" (ken). La Constitution érige en principe l'autonomie locale. Après la fin de l'Occupation, Tokyo a travaillé à remettre les pouvoirs locaux en tutelle, mais le principe subsiste - avec l'élection des maires et des gouverneurs au suffrage universel, l'interdiction absolue du cumul des mandats et le transfert de l'essentiel des recettes fiscales aux ken et aux communes. Les citoyens peuvent obliger leur maire à démissionner par pétition, présenter des résolutions aux conseils municipaux ou régionaux, demander par ce biais l'organisation de référendums locaux et solliciter des inspections de l'administration centrale sur la gestion municipale 15.

Les mouvements de contestation peuvent aussi réutiliser le fond culturel de la communauté villageoise traditionnelle (mura), qui reste enraciné dans l'inconscient collectif comme la matrice de toute l'organisation sociale. Cette culture participe autant du principe "d'horizontalité" (égalitarisme, démocratie de base) que de la "verticalité" (hiérarchie, autorité) à laquelle certains ont

14. Ce passage se fonde sur les interviews de M. Muraoka Isotsugu, directeur exécutif de Green Coop-Bloc Nord (1er octobre 1996) et de M. Ikeuchi Makoto, directeur exécutif du Club de Hokkaïdo (15 octobre 1996). La thèse de LaM Peng-Er, "The Network Movement and the Old Politics Parties in Urban Japan" (Columbia University, 1992) m'a été d'une grande utilité. Les données chiffrées sont extraites de Data han seikatsu kurabu (Registre de données sur les Clubs de la vie) d'août 1996 ; pour Green Coop, de la brochure Watashi wa Green Coop desu (Nous sommes Green Coop), édité par la coopérative en 1996 et de Green Coop no goannai (Guide de Green Coop), août 1996.

15. Sur les pouvoirs locaux, le livre de référence est celui de Kurt STEINER, Local Government in Japan, Stanford : Stanford University Press, 1965. En français, JeanMarie Bourssou, "Des communautés locales autonomes à $30 \%$ ", Annuaire des Collectivités Locales, GRAAL (CNRS), 1983, pp. 80-107. 
voulu abusivement la réduire 16 . Les contestataires peuvent reprendre à leur manière les modèles d'organisation du mura et même parfois s'appuyer sur les organisations de base entretenues par les autorités, comme les chônaikai (associations de voisinage) 17.

Historiquement, la mouvance alternative japonaise est issue de deux courants. Le premier est constitué par les "mouvements de citoyens" (shimin undô) et les "mouvements d'habitants" (jûmin undô) des années 1960-197018. Les premiers, en liaison avec l'opposition de gauche, se sont illustrés dans le mouvement anti-atomique, la lutte contre le Traité de sécurité avec les EtatsUnis et la guerre du Vietnam. Les seconds, méfiants à l'égard des partis, avaient des objectifs souvent limités à la vie quotidienne locale, notamment la lutte contre la pollution. Les uns et les autres ont connu une éclipse marquée dans les années 1980, où triomphe un "conservatisme de confort" soutenu par la prospérité matérielle.

D'autres groupes, moins connus, ont été dès leur apparition porteurs d'une conception alternative globale de la démocratie et de la vie sociale. Ils se distinguent aussi bien des shimin undô, par leur refus de toute affiliation avec la politique classique, que des jûmin undô, dont l'action est par essence ponctuelle. Ce sont essentiellement des coopératives de consommation autogérées. Avec leur prolongement politique (le NET), elles représentent aujourd'hui la tentative la plus aboutie pour réinventer la démocratie à partir de l'activité micro-citoyenne et des préoccupations quotidiennes. Après une longue période d'implantation obscure, elles se trouvent projetées sur le devant de la scène par la crise des formes classiques de la participation politique.

\subsection{Seikatsu Kurabu et Green Coop : historique et organisation}

Les Seikatsu Kurabu (Clubs de la vie) regroupent aujourd'hui 17 coopératives régionales 19 et 234368 foyers-membres. La plus importante est celle de

16. Le meilleur exemple de cette réduction est fourni par le livre célèbre de Chie NAKANE traduit en français sous le titre La société japonaise, Paris : Armand Colin, 1974. Pour l'illustration de l'autre tradition, voir Yoshio SUGIMOTO and Ross MOUER, Images of Japanese Society, London \& New York : Kegan Paul, 1989.

17. Sur ces structures, voir Theodor BESTOR, Neighborhood Tokyo, Tokyo : Kodansha, 1989.

18. Sur ces mouvements, voir notamment Kurt STEINER, Ellis KRAUSS and Scott FlaNAGAN, dirs., Political Opposition and Local Politics in Japan, Princeton University Press, 1980, (Part III: "Citizens' Movements", pp. 187-313). En français, Jean-Marie Bouissou, Le Japon depuis 1945, Paris : Armand Colin, 1992, pp. 63, 68, 95-98, 139 141 et $170-172$.

19. D'après la loi japonaise, pour protéger les intérêts des chaînes de grande distribution, une coopérative de consommation ne peut pas déborder le cadre d'un département (ken). 
Kanagawa-ken, avec 48175 membres. Le premier Club a été créé en 1965 à Tokyo par Iwane Kunio, un militant socialiste formé dans les shimin undô. Après le scandale du lait Morinaga contaminé à l'arsenic, il commence par distribuer du lait naturel pour un petit groupe de 200 membres. En 1968, la coopérative adopte sa structure caractéristique, le han. En 1971, un syndicaliste des chemins de fer, Yokota Katsumi, crée le Club de Kanagawa.

Les années 1970 sont celles de l'enracinement autour de Tokyo, à Saïtama (1975), Chiba (1976) et Nagano (1977). En 1982, les Clubs atteignent 100000 foyers-membres. Ils essaient ensuite de s'implanter dans le Nord (Hokkaiido, Miyagi, Yamanashi, Iwate, Tochigi) et le Japon central (Shizuoka, Aïchi), mais sans grand succès. Sauf à Hokkaïdo, les nouveaux Clubs stagnent à moins de 5000 membres et plus de $85 \%$ des effectifs restent concentrés dans la mégapole tokyoïte. Dans le Japon central, les Clubs se heurtent aux coopératives du Kansaï, les plus anciennes (Coop Kobé a été fondée en 1921) et les plus solides du Japon, très influencées par les militants communistes. Ils doivent se contenter d'une alliance avec deux petits groupes, Ôsaka seikyô jiyû rengô (Fédération des coopératives libres d'Osaka) et Shutoken Coop rengô (Fédération des coopératives métropolitaines) à Tokyo. Mais dans les neuf préfectures du Sud-Ouest, ils trouvent un allié de poids avec Green Coop, qui $s$ 'inspire d'idéaux très semblables aux leurs.

L'origine de Green Coop remonte à quatre coopératives créées par les étudiants de Fukuoka dans la foulée du mouvement de 1968 pour fournir des produits alimentaires et des fournitures scolaires, qui se sont ouvertes ensuite à l'ensemble de la population. Ses "pères fondateurs" sont Muraoka Isotsugu, un salarié de Kyûdai (Université de Kyûshû), et un professeur, Takeda Keijirô (1925-1994), qui est l'idéologue du mouvement 20 . En 1988, 25 coopératives de l'Ouest du Japon organisent une centrale d'achat commune: Green Coop est née. Elle est présente dans les neuf ken de Hiroshima à Kagoshima. De 140311 foyersmembres en 1988, elle est passée à 256524 en 1995, dont 138000 dans le Fukuoka-ken et 55000 à Kumamoto. Mais elle aussi se heurte au bastion des coopératives classiques dans le Japon central ; elle ne parvient pas à remonter à l'est de Hiroshima, où sa présence reste d'ailleurs symbolique (2700 membres).

Pour 1995-1996, le chiffre d'affaires de Green Coop a dépassé 52 milliards de yen. Les Seikatsu Kurabu ont un peu moins de membres, mais un chiffre d'affaires de 76,3 milliards de yen. Les Clubs se classent au $9 \mathrm{e}$ rang des 669 coopératives japonaises pour le nombre de membres et au $8 \mathrm{e}$ pour le chiffre d'affaires, Green Coop se situant au $8^{\mathrm{e}}$ pour les membres et au $11 \mathrm{e}$ pour le chiffre. Mais ils restent loin derrière Coop Kobé, la plus ancienne et la plus puissante des coopératives, qui dépasse un million de membres et 327 milliards de yen de chiffre d'affaires 21 .

20. Le recueil de ses pensées a été publié sous le titre de Kyôsei (Symbiose) en 1995 par Green Coop.

21. Cf. Co-Op Facts and Figures, Tokyo : Zenkoku seikyô rengôkai (Fédération japonaise des coopératives), 1990. 
Avec Ôsaka seikyô et Shutoken Coop, les coopératives alternatives se sont regroupées en 1989 en créant Alternative Japan, qui est avant tout une centrale commune pour leurs achats et leur action à l'étranger. Les traits qui les distinguent fondamentalement des coopératives classiques sont l'autogestion de leur structure, leur volonté de recruter des militants et non de simples clients, de proposer un modèle alternatif global pour la vie quotidienne et, au-delà, de "passer au politique".

\subsection{Les déclinaisons de l'autogestion}

\section{* L'autogestion de la structure de distribution}

Par principe, les Clubs et Green Coop n'ont pas de boutiques. Leur esprit est celui de la géographie mobile du "réseau" (nettowâku), mot-clé de tout le discours alternatif. La distribution se fait par tournées hebdomadaires de camionnettes. L'unité de base (han) est formée de cinq à dix foyers voisins, dont chacun groupe à tour de rôle les commandes et les paiements. Les Clubs en ont 27 757. Dix han forment un district (chiku), dix districts forment une branche (shibu). La plus grande unité comprend donc environ 5000 foyers-membres (Green Coop en a 55). Chaque branche a un entrepôt géré par des volontaires, quelques salariés (chauffeurs-livreurs) et une salle de réunion. Les districts servent de courroie de transmission et à renforcer le sentiment d'appartenance par de multiples activités conviviales. Au-dessus, on trouve des centres de groupage des achats, qui peuvent desservir plusieurs ken (Green Coop en a trois).

Le han incarne les principes de la "démocratie de base" qui inspirent toute l'organisation. Chaque niveau est animé par un comité (iinkai) élu par les responsables des han. Celles-ci 22 se réunissent chaque mois en assemblée de chiku et, une fois par an, en assemblée générale de branche pour désigner le comité de shibu. Cette désignation se fait sans procédure définie : l'idéal est que cela se fasse "naturellement" (nantonaku). La rotation est de règle, tous les deux ou trois ans. Les responsables ne sont jamais rétribuées, et certains Clubs, comme Hokkaïdo, refusent même de les défrayer. Le recours aux salariés, contraire aux principes, est limité au minimum : les Clubs n'en ont que 952.

C'est dans le même esprit que les coopératives alternatives font payer un droit d'entrée, alors que les autres ouvrent leurs magasins à tous sans condition. Leurs adhérents doivent être des militants, qui font consciemment le choix d'un mode de vie. A Green Coop, l'adhésion (2 000 yen : environ 90 F) et les cotisations mensuelles ( 500 yen : moins de $25 \mathrm{~F}$ ) sont très bon marché. Mais à Kanagawa, le droit d'entrée au Club atteint 230000 yen (étalés par versements mensuels de 1000 yen), en sus d'une cotisation mensuelle de

22. Il s'agit sans exception de femmes (voir infra). 
1000 yen. Notons que l'autonomie de chaque Club est la règle, sur ce plan comme sur tous les autres. A Hokkaïdo, les droits sont semblables à ceux de Green Coop. La liberté des membres est strictement respectée : les droits payés sont remboursés à ceux qui décident de quitter le groupe.

\section{* L'autogestion de la communauté locale}

Au-delà de leur réseau, les coopératives alternatives cherchent à promouvoir "une société coopérative de petites communautés" (kumiai chiiki shakai), qui est présentée comme l'antidote à la "société verticale" du Japon conservateur. A cette fin, ils développent des organisations annexes qui visent à prendre en charge la vie quotidienne de plus en plus largement.

L'outil de base est le worker's collective 23. Ces "collectifs" groupent quelques dizaines de ménagères, qui se cotisent pour réunir le modeste capital nécessaire et se partagent le travail. Les Clubs en comportent 267. Green Coop n'a créé le premier qu'en 1994 et n'en avait encore que 11 en 1996. Ils proposent surtout des services de proximité : aide ménagère aux personnes âgées et malades, crèches, livraison de repas à domicile. D'autres font de la récupération et du recyclage, du petit artisanat et des activités culturelles. Mais, si leur importance théorique est grande, leur activité reste modeste. On est encore loin de la communauté alternative idéale dépeinte par $\mathrm{K}$. Yokota, où ils offriront, dans une optique non lucrative, la gamme complète des services de proximité et « des ateliers de réparation des appareils électriques, des boulangeries, des salons de coiffure, des cordonneries, des pressings et des garages » 24 .

Les Clubs et Green Coop ont aussi commencé à offrir des services nouveaux, sous le vocable général de fukushi (bien-être). Le Club de Kanagawa a ouvert une maison pour handicapés dès 1977 et un Fukushi Kurabu en 1989. Green Coop a créé en 1994 le Fukushi rentai shikin (Fonds de bien-être), financé par une augmentation de 100 yen $(4,80 \mathrm{~F})$ de la cotisation mensuelle. Il se consacre aux handicapés et aux personnes âgées, auxquelles il propose des "centres de jour" pour se retrouver et continuer à mener une vie sociale. Green Coop a aussi une mutuelle d'assurance (48 000 adhérents en 1995) et même un service de pompes funèbres.

L'idéal, là encore, est de soustraire à la logique capitaliste les secteurs essentiels pour la vie quotidienne que sont « le logement, l'épargne et le crédit, les services de santé » 25 . Si les alternatifs sont encore loin du compte, leur obstination atteste la détermination de leur stratégie d'avancée pas à pas.

23. Pour lesquels il n'existe pas de mot propre en japonais ; c'est toujours l'expression anglaise qui est utilisée.

24. YoKota Katsumi, I among Others, An Introspective Look at the Theory and Practice of the Seikatsu Club Movements, Yokohama : Seikatsu Club Seikyô Kanagawa, cité par LAM Pen-Er, op. cit., p. 93.

25. Ibid. 
* Le refus de la centralisation

Autogestion oblige : chaque coopérative régionale doit être le plus autonome possible. Les quatre coopératives alternatives ont une centrale d'achat commune pour les produits importés (sucre, bananes), mais chaque groupe régional recourt surtout à ses fournisseurs locaux. Les Clubs ont un organe central de 24 membres élus, le Rengôkai rijikai (Assemblée des délégués) et deux centres de recherche "sur la vie du secteur citoyen" (Shimin sekutâ seikatsu kikko) et "sur les mouvements sociaux" (Shakai undô kenkyû sentâ). Mais leurs fonctions sont réduites à des réflexions générales, diffusés par la revue Seikatsu to Jichi ("Vie et Autonomie").

\subsection{Autres éléments d'une idéologie alternative}

\section{* Le féminisme}

Green Coop et les Clubs se flattent « d'agir naturellement du point de vue des femmes », puisque leurs fonctions relèvent du champ d'activité des ménagères. Les femmes sont aussi porteuses de valeurs spécifiques (attention aux autres, dévouement, pacifisme) dont la société traditionnelle ne permet pas l'épanouissement. C'est sur elles que repose le fonctionnement des réseaux et elles doivent par principe assurer les fonctions de direction. De fait, les responsables des chiku et des shibu sont toujours des femmes. Mais partout, les pèresfondateurs ont été des hommes. Si Iwane à Tokyo et Yokota à Kanagawa se sont retirés, beaucoup d'autres sont encore actifs, en général au poste discret de directeur exécutif (senmu riji), comme M. Muraoka Isotsugu à Fukuoka ou M. Kobayashi Shigenobu à Hokkaïdo. Partout où nous avons enquêté, c'est un homme qui a répondu à l'interview, hors de la présence de tout élément féminin. On peut aussi noter qu'il n'y a que trois femmes pour neuf hommes parmi les conseillers en titre (komon) du "Fonds de bien-être" de Green Coop et 10 hommes sur 24 délégués au Rengôkai rijikai des Clubs. Même placé sur les marges de l'organigramme, l'élément mâle pèse toujours lourd dans le mouvement.

\section{* L'écologie}

Chaque coopérative assure elle-même l'essentiel de ses approvisionnements au niveau local. Les contrats limitent l'usage des engrais et pesticides chimiques, en interdisent certains et imposent une obligation de transparence. Mais cette démarche se veut souple et éducative. D'après le responsable du Club de Hokkaïdo, « pour le moment, il s'agit de convaincre peu à peu les agriculteurs de changer de méthode ». La récupération et le recyclage sont un élément indispensable de la panoplie alternative. La mémoire collective du mouvement révère l'exemple du Club de Kanagawa, dont l'essor initial s'est 
fondé sur une campagne contre les détergents chimiques (1980). En pieux souvenir, chaque coopérative pratique le recyclage des vieilles huiles de friture en savon "naturel", outre la récupération du verre, du papier et des boîtes à œufs en plastique. La lutte anti-nucléaire est également un must absolu, qui marie l'écologie aux souvenirs historiques et au profond tropisme pacifiste du peuple japonais.

On peut également ranger sous la rubrique "écologie" la défense des minorités ethniques, là où il en subsiste. A Hokkaïdo, le mensuel du Club s'intitule Chuppu ("Le Soleil"), dans la langue des Aïnus, la population aborigène réduite aujourd'hui à moins de 40000 personnes.

\section{* L'internationalisme}

Dès 1982, les Clubs ont envoyé cinq représentants à la Conférence de l'ONU sur le désarmement. Alternative Japan a participé au forum des ONG féminines à Pékin en 1995. En Corée, l'organisation d'une coopérative alternative baptisée "Vie précieuse" (Hansalin) est en cours, grâce à l'envoi d'experts et à la formation de militants locaux. Aux Philippines, la coopération se fait avec les paysans des îles Negroes, auxquels Alternative Japan achète sucre et bananes, mais qu'elle aide aussi à se reconvertir (stages au Japon, envoi d'experts). De même, des excursions de lycéens japonais doivent contribuer à une prise de conscience des problèmes du Tiers-Monde. Quant au Club de Hokkaiido, il a reçu un "Nobel alternatif" et il a été la seule ONG japonaise parmi les cinquante distinguées lors du Cinquantenaire des Nations Unies. Ce qui suppose une politique active de candidature...

\section{* La cyber-démocratie}

Nos interlocuteurs ont souvent présenté la communication informatique comme le moyen d'action le mieux adapté au paradigme organisationnel du mouvement alternatif : le réseau d'individus autonomes capables de contacter tous ceux qui pensent comme eux pour organiser leur synergie... Mais ni à Fukuoka, ni à Hokkaïdo, ils n'étaient présents sur Internet en 1996. Au niveau central, seul Alternative Japan possède son site. Ce retard peut s'expliquer par la sociologie des militantes, dont la plupart sont des ménagères de plus de 40 ans, mal à l'aise devant l'informatique. Mais on peut aussi y voir l'effet d'un certain passéisme, qui sur-valorise la chaleur du contact humain et la convivialité de la petite communauté. De fait, informatiser les commandes signifierait la fin du han... Les plus actifs en la matière sont les écologistes d'Oita, qui ont été à l'origine du mura okoshi undô 26. 
Leur serveur New Coara (http://www.@fat.coara.or.jp.) a diffusé en quasi direct la rencontre du président coréen et du premier ministre japonais le 25 janvier 1997 - exercice symbolique de démocratie directe 27 . À Fukuoka, le mouvement contre l'aménagement de la baie de Wajiro a créé son site (http://www.bekkoame.or.jp.) en 1996.

\section{LE PASSAGE AU POLITIQUE : LE NET}

\subsection{Le "pari gramscien" et ses nuances}

L'objectif ultime des fondateurs des Clubs et de Green Coop était politique. Iwane appartenait à l'aile droite du Parti socialiste, qu'il ne quittera qu'en 1977. Il partage avec Yokota une source d'inspiration commune, la pensée de Gramsci. Lam Peng-Er cite de nombreux articles qu'ils lui ont consacrés et nos interlocuteurs n'ont jamais hésité à reconnaître la filiation idéologique gramscienne. Ils lui ont emprunté la théorie sur la formation d'une "culture alternative", fondée sur l'offre de pratiques non capitalistes de production, de distribution et de consommation, qui permettra d'établir une "interaction citoyenne au quotidien", organisée selon des normes nouvelles qui feront peu à peu changer le système des valeurs au niveau local. Il naîtra ainsi des communautés d'un type nouveau. Le mouvement gagnera de proche en proche, jusqu'à subvertir in fine le niveau national.

A cette fin, les Clubs et Green Coop multiplient conférences, festivals et excursions éducatives. Leurs maisons d'édition diffusent revues et bulletins par millions d'exemplaires. Les membres sont d'ailleurs réellement concernés : d'après Lam Peng-Er, $45 \%$ d'entre eux assistent aux conférences, non sans effet, puisque si seulement $42 \%$ de ceux qui entrent aux Clubs se disent "intéressés par la politique", le pourcentage grimpe à $64 \%$ chez les adhérents confirmés.

26. Sur ce mouvement très ambigu, à cheval entre mouvance alternative et aménagement technocratique du territoire "relifté" à l'échelle humaine, voir Jean-Marie BouIssou, "Un nouveau Japon ? Regards sur les mouvements alternatifs", Les Études du $C E R I, \mathrm{n}^{\circ} 30$, juillet 1997 , pp. 30-35. Il n'existe pas de littérature en langue occidentale sur ce mouvement. En japonais, HIRAMATSU Morihiko, Tôkyô de dekinai koto o yattemiyô (Faisons ce que l'on ne peut pas faire à Tokyo), Tokyo : NESCO, 1987 ; MARUTANi Kaneyasu, Isson ippin pâfomansu (La mise en ouvre du mouvement "un village, un produit), Tokyo : Hokuto Shuppan, 1987. Voir aussi John KNIGHT, "Town-making in Rural Japan : An Example from Wakayama", Journal of Rural Studies, Vol. 10 (3), 1994, pp. 249-261 et "Making Citizens in Postwar Japan", in Chris Hann and Elizabeth Dunn, dirs., Civil Society. Challenging Western Models, London : Routledge, 1996, pp. 222-241.

27. Asahi Shinbun du 23 janvier 1997. 
La "société coopérative de petites communautés" est le concept de base commun à tous les groupes. Mais les nuances sont nombreuses. Green Coop met au centre de son discours la notion de "symbiose" (kyôsei), qui figure dans le titre de son mensuel Kyôsei no jidai ("L'ère de la symbiose"). Les pensées de son fondateur (cf. le recueil Kyôsei) proclament la nécessité d'une quadruple symbiose: entre l'homme et la nature (hito to shizen), entre tous les humains (hito to hito), entre la femme et l'homme (onna to otoko) et entre les pays du Sud et ceux du Nord (minami to kita). On y perçoit la nostalgie d'un "Âge d'Or" qui se décline sur le thème de la communion avec la nature, de la convivialité, des "vieillards aimés", pris en charge sur le mode quasi-familial, et de la fin de tous les conflits. Mais les vieux Clubs de la région de Tokyo privilégient la thématique plus politique de l'"autonomie" (jichitai), qui vient en droite ligne du discours des partis de gauche pendant "l'ère des gouvernements locaux progressistes", quand socialistes et communistes gouvernaient la majorité des mégapoles du Japon (1967-1976 environ). De son côté, le Club d'Hokkaïdo prône la disparition de la notion même de "communauté", dénoncée comme un héritage confucéen imprégné d'autoritarisme, pour recentrer la société sur la notion occidentale d"individu". Le han lui-même, nécessaire pour la phase initiale d'organisation, devra disparaître in fine. Une perspective qui horrifierait les autres Clubs!

Ces nuances s'enracinent dans l'histoire de chaque groupe. Green Coop s'est développé par fusions, alors que les Clubs ont rayonné à partir d'un centre unique. Et à Hokkaïdo, on souligne l'originalité de l'île, terre de pionniers et de marginaux...

\subsection{Le NET 28}

Pour concrétiser leur projet politique en marge du jeu des partis classiques, les coopératives alternatives ont donné naissance, dès 1979, au mouvement NET (network), destiné à pénétrer les assemblées locales. NET et coopératives ont des organisations parallèles (han, chiku...), mais jamais confondues, car la Loi sur les coopératives (Art. 2) leur interdit « d'être utilisées par un parti politique quelconque ». Les premiers succès électoraux du NET, à partir de 1982, ont d'ailleurs provoqué une tentative (manquée) du PLD pour durcir cette loi en 1985-1986.

28. Ce passage se fonde essentiellement sur les données fournies par le mensuel Jikkatsu kurabu ("Le Club de la vie autonome") du 24 juillet 1996 et sur les interview's de Mmes Utsugi Tomoko, conseillère municipale du NET à Yamatoshi (Kanagawa-ken) en 1992, Komiya Fumiko et Imamura Emiko, conseillères municipales du NET à Fukuoka (4 octobre 1996), Shichiri Tomiko, secrétaire générale de Sapporo-NET ( 9 octobre) et Nakajima Kazuko, conseillère municipale du NET de Sapporo et membre de la direction centrale du NET-Japon. Également, l'indispensable thèse de Lam PenEr. 
En 1996, les NET sont présents dans 9 départements (Hokkaïdo, Iwate, Yamanashi, Chiba, Saïtama, Tokyo, Kanagawa, Nagano et Fukuoka). Ils ont 109 sections et 6700 adhérents. Ils comptent 123 élues (75 en 1992) qui siègent dans 69 assemblées locales, dont deux conseils généraux (3 à Tokyo, 4 à Kanagawa) et cinq cités à statut spécial (shiteitoshi) : Sapporo (3 élues), Chiba (4), Yokohama (6), Kawasaki (4) et Fukuoka (2). A Tokyo, ils sont représentés dans 10 des 23 arrondissements et 20 communes de banlieue. A Kanagawa, outre le conseil général, Yokohama et Kawasaki, ils sont présents dans 15 conseils municipaux.

Le NET se définit comme un mouvement de présentation de candidatures (dairinin undô) et non comme un parti. Ses élues sont des "amateurs", qui doivent abandonner leur siège après deux mandats et reverser leurs indemnités au mouvement. Elles refusent le titre de sensei (maître) dont sont affublés les élus japonais. Elles dénoncent « la politique où on sollicite humblement le maître » (sensei onegaishimasu yo seiji), et prônent « une politique où les gens participent activement »(sankagata seiji). Elles s'attachent par priorité aux problèmes de vie quotidienne : l'hygiène des cantines, l'écologie dans les jardinets des écoles maternelles, l'aménagement des rues pour les handicapés, le recyclage des ordures. Elles traquent le sexisme jusque dans les cahiers d'appel à l'école, où les filles figurent après les garçons. Mais elles luttent aussi contre le nucléaire et les nuisances provoquées par les bases militaires ainsi que pour les droits des immigrés. Elles contestent les projets "tout béton" d'aménagement urbain, au risque de représailles musclées 29 . Enfin, elles relaient les procédures intentées pour obtenir la transparence administrative (jôhô $k \hat{o} k a i)$, un thème sur lequel les citoyens manifestent aujourd'hui une grande pugnacité.

Les NET ont attendu 1993 pour créer un appareil central, le Kanjikai (Comité des responsables) de huit membres. Les kanji, désignés par chaque NET régional, se réunissent cinq ou six fois par an. Chaque section envoie aussi deux ou trois déléguées à une assemblée générale bi-annuelle (Chiiki NET katsudô kôryû chûkai). Le Dairinin undô kôrŷ̂ sentâ (Centre d'échanges sur le mouvement de présentation de candidatures) sert de base de données et publie une revue trimestrielle. Mais aucun de ces organes n'a vocation à imposer une "ligne politique". Aux législatives du 20 octobre 1996, par exemple, les responsables centraux penchaient pour un soutien au Parti démocrate (Minshutô), mais chaque NET est resté libre d'accorder ou non sa recommandation (suisen) à un candidat. La plupart s'y sont refusés.

29. A Fukuoka, une conseillère opposée au projet de la baie de Wajiro a été menacée en pleine séance par le vice-président du Conseil municipal : « Si vous vous obstinez, vous ne pourrez plus dormir la nuit », et sa voiture a été dégradée. Wajiro Higata tsîshin ("Le courrier des laisses de Higata"), n³9, juillet 1996, p. 2. 


\subsection{Ambiguiités}

Si le mouvement des coopératives alternatives semble défier l'ordre politique établi et la culture autoritaire dominante, il n'en reste pas moins ambigu sous nombre d'aspects.

Les han des Clubs et de Green Coop rappellent de très près les tonarigumi de l'époque Tokugawa, qui regroupaient une dizaine de foyers et assuraient le contrôle policier, et les "associations de voisinage" (chônaikai) semi-officielles qui les ont remplacés après 1945. Cette structure est aussi celle de nombreuses sectes et des machines électorales (kôenkai) des politiciens classiques 30 . Le fait que les alternatifs se coulent dans ces modèles hérités des communautés traditionnelles et en réutilisent la symbolique (convivialité, attachement au territoire) est d'autant plus ambigu que le système de valeurs de ces communautés ne reconnaît à l'individu qu'une "identité contextuelle", définie par sa place sociale et les obligations qu'elle commande. Une conception fort éloignée de la primauté de l'individu en tant que tel, qui est la valeur cardinale de la démocratie occidentale.

On peut aussi s'interroger sur la focalisation obsessionnelle des coopératives sur le problème du vieillissement. Outre le "Fonds de bien-être", Green Coop propose une vaste gamme de produits pour le 3e âge, du déambulateur ergonomique aux couverts spéciaux pour arthritiques. Cette focalisation se retrouve dans les interviews de leurs responsables. La majorité des adhérents n'ont pourtant qu'entre 35 et 45 ans. Mais ce sont eux dont les parents pâtissent aujourd'hui du manque d'institutions pour les vieillards et qui parviendront à la retraite vers 2020, quand le Japon n'aura plus que deux actifs pour un inactif. Ils sont donc très sensibles au discours sur "la société vieillissante", dont le PLD a fait une angoisse nationale, qui lui a d'ailleurs permis de réviser la protection sociale à la baisse. Force est de constater que ce discours et celui des alternatifs se répondent. Et quand ceux-ci prennent en charge les personnes âgées, ils aident de fait le gouvernement en palliant des lacunes du service public.

Une conjonction encore plus troublante entre le discours dominant et celui des alternatifs est l'usage intensif du terme kyôsei. Depuis quelques années, de l'extrême-droite jusqu'à la gauche, on ne parle plus de l'ordre social, économique ou international à réinventer autrement que comme "symbiose". Nous avons analysé ailleurs les raisons de l'émergence de ce concept ubiquiste et l'usage qu'en font les élites 31 pour essayer de désamorcer les tensions au sein d'une société ébranlée par la mondialisation. Le fait que nombre d'alternatifs le reprennent à leur compte fait évidemment problème...

30. Sur les kôenkai, voir Jean-Marie Bouissou, "La convivialité comme remède au conflit. Les 'associations de soutien' des politiciens japonais", in Jean-Marie Bouissou, dir., L'envers du consensus..., op.cit., pp. 75-89.

31. Jean-Marie Bouissou, "Penser l'autre en se pensant soi-même : le Japon face à l'Asie", in David Camroux et Jean-Luc Domenach, dirs., La réinvention de l'Asie, Paris : Le Seuil, 1997, pp. 301-326. 
Green Coop et les Clubs sont aussi de farouches défenseurs de l'agriculture japonaise. En 1993, ils ont créé DEVANDA 32, qui compte plus de 10000 groupes à travers le Japon. Son objectif est d'accroître l'autosuffisance alimentaire et d'organiser la chaîne producteur/consommateur dans une optique écologique. En 1994, DEVANDA a organisé une pétition nationale contre les importations agricoles. Il invoque à la fois l'hygiène (les produits étrangers contiendraient trop de produits chimiques) et l'autogestion ("Manger ce qu'on produit soi-même"). Mais là aussi, on voit s'esquisser d'étranges connivences avec le lobby rural du PLD, le plus rétrograde...

À terme, ces ambiguïtés pourraient faciliter la récupération du mouvement par des élites qui ont déjà prouvé leur capacité à intégrer les nouveaux entrants sur le marché politique. Elles se retrouvent dans un autre cas d'école - le mouvement antipollution de Nishiyodogawa - qui illustre comment l'idéologie alternative tend aujourd'hui à devenir dominante au sein des mouvements de lutte traditionnels, genre jûmin undó.

\section{QUAND L'ESPRIT ALTERNATIF VIENT AUX LUTTES LOCALES : L'EXEMPLE DE NISHIYODOGAWA 33}

\subsection{Nishiyodogawa, phase 1 : la lutte défensive}

C'est en 1968 que M. Moriwaki Kimio, militant communiste, commence à organiser les victimes de la pollution à Nishiyodogawa- $k u$, à l'ouest d'Osaka. L'arrondissement, situé dans une cuvette, compte environ 100000 habitants. Stations thermiques et hauts-fourneaux y engendrent une pollution au dioxyde d'azote (NO2) telle que "les oiseaux tombaient du ciel". En 1974, 4,5\% de la population sera certifiée comme souffrant de maladies respiratoires. Aujourd'hui encore, Osaka compte 18890 des 101258 victimes "officielles" de la pollution indemnisées par l'administration.

Après 1970, les premiers succès des victimes des "Quatre grands cas" 34 encouragent les militants de Nishiyodogawa. En 1972, Moriwaki crée l'Association des victimes de la pollution et de leurs familles. En 1973, celle-ci orga-

32. Do it Eco-Vital Action Network for Agri-native (sic).

33. Le passage ci-après se fonde sur les interviews de MM. Kasagi Hiroo, directeur des recherches de la Fondation pour la restauration des zones polluées du Japon et secrétaire général de Blue Sky Society, et Moriwaki Kimio, directeur de la Fondation précitée et secrétaire général de la Commission pan-japonaise d'action pour les victimes de la pollution (18 septembre 1996).

34. C'est-à-dire les quatre affaires qui font éclater les scandales de pollution à la fin des années 1960 : empoisonnement par le mercure de Minamata et de la rivière Agano, contamination par le cadmium dans la région de Toyama, asthme de Yokkaïchi. 
nise un sit-in permanent devant la mairie d'Osaka. Le gouvernement local est passé aux progressistes en 1971. Son appui va être décisif. Il offre un programme d'avant-garde pour recenser les victimes de la pollution et les faire indemniser par des contributions des entreprises locales. Fort de ce succès, le mouvement communiste organise un bureau de liaison national (1978), puis l'Association japonaise des victimes de la pollution atmosphérique, ou JAPA (1981), forte de plus de 100000 adhérents, dont Moriwaki devient directeur général. Sous la pression, le PLD vote une loi inspirée des mesures prises à Osaka (1974). L’Association de Nishiyodogawa a été consultée : «Nous étions appelés à Tokyo presque chaque jour pour rencontrer les fonctionnaires et discuter avec eux chaque mot du nouveau programme » 35 .

Mais après 1976, les gouvernements locaux progressistes tombent partout, y compris à Osaka. Le PLD relâche aussitôt les standards qui limitaient les émissions de $\mathrm{NO}^{2}$. La JAPA répond par une vague d'actions en justice. À Nishiyodogawa, trois procédures sont intentées contre neuf entreprises. Les firmes résistent avec détermination. Il faut plus de dix ans pour que les plaignants l'emportent en première instance à Chiba (1988), Nishiyodogawa-1 (1991), Kawasaki (1994) et Kuraishi (1995). Mais le contexte change. Le PLD chancelle et scissionne en 1993. Le "triangle d'airain" qui l'unissait au patronat et à l'administration perd sa cohésion. La spéculation et la crise attisent le mécontentement contre le système économique. Le patronat juge prudent de transiger. Kawasaki Steel traite avec les plaignants de Chiba. En 1995, deux semaines avant le prononcé du jugement dans les cas Nishidogawa-2 et -3 , les firmes visées reconnaissent leur responsabilité. Leurs présidents viennent s'incliner devant les victimes. Elles payent 2,5 milliards de yen aux plaignants et 1,5 milliard de yen à une Fondation pour restaurer l'environnement à Nishiyodogawa, créée en 199136.

\subsection{Nishiyodogawa, phase 2 : le projet alternatif}

Avec cette victoire, le mouvement passe à une phase nouvelle. Le but est désormais d'organiser "le renouveau de la communauté" (chiiki kaiseikai) grâce aux fonds obtenus. Une autre génération de militants entre en scène, dont le prototype est M. Kasagi Hiroo. Quand le communiste Moriwaki organisait les malades de Nishiyodogawa, le "soixante-huitard" Kasagi luttait pour préserver le quartier historique de Nakanoshima, avec un groupe d'intellectuels baptisé Conseil pour l'environnement urbain. Ils y parviendront en créant une fête populaire (Nakanoshima matsuri), organisée chaque mai par des comités

35. Kimio MORIWAKI, "The experience of organizing pollution victims in Nishiyodogawa”, travaux du colloque Japan's Pollution Experience, miméo, p. 2.

36. A. Muramatsu, “Japan's air pollution court cases”, ibid., p. 15. 
de citoyens (shimin dantai), qui est devenue un événement majeur, avec 300000 participants en 1996.

La culture des deux mouvements est très différente. Selon un témoin, dans les années 1970, «Moriwaki et Kasagi ne pouvaient même pas se parler ». D'un côté, il y avait « la rude campagne des victimes de la pollution » et de l'autre « le sociable mouvement citoyen pour le développement de la communauté »37. Les premiers contacts n'ont lieu qu'en 1990. Mais la fin de la bataille judiciaire et le vieillissement de l'Association (1'âge moyen des victimes dépasse $72 \mathrm{ans}$ ) ont amené ses responsables à conclure qu'il leur fallait du sang neuf. Le recul du PCJ y est peut-être aussi pour quelque chose (entre 1979 et 1993, il est tombé de $10,4 \%$ des voix et 39 élus à $7,8 \%$ et 15 élus). En 1991 , les deux groupes présentent en commun un "plan de base" pour la restauration de Nishiyodogawa, puis deux autres en 1994 et 1995. Le mouvement chiiki kaiseikai amène avec lui des jeunes gens frais émoulus de l'université, qui forment l'essentiel des permanents de la Fondation, dont Moriwaki et Kasagi sont respectivement président et directeur des recherches.

\subsection{Ambiguïtés}

La Fondation constitue clairement un mouvement d'un type nouveau, qui transcende la lutte défensive des victimes de la première génération. Elle ambitionne de bâtir un environnement sans pollution, en enfouissant la circulation et en réhabilitant le transport par eau. Elle veut faire "autogérer l'environnement" par les habitants. Ceux-ci mesurent en permanence le taux de $\mathrm{NO}^{2}$ dans l'air, grâce à un équipement très simple, des filtres dans de petites éprouvettes que la Fondation collecte et analyse. Pour inscrire son action dans la durée, celle-ci forme les enfants grâce à des forums de discussion dans les radios locales. Enfin, elle veut faire de Nishiyodogawa un modèle pour les zones polluées "d'Asie et d'ailleurs", où l'on formerait des experts dans une université spécialisée et dont l'expérience se partagerait via Internet...

On ne peut pourtant qu'être frappé des connotations nostalgiques et passéistes que véhicule le discours de la Fondation sur la culture commmunautaire locale. « La pollution (...) a volé aux gens plus que leur santé : leur culture, leurs communautés et la joie de vivre du temps où les enfants jouaient dans les petits canaux et attrapaient des poissons (...) et où les gens se parlaient d'un bord à l'autre en suspendant le linge à sécher le long des rives ». C'est ce mode de vie convivial qu'il s'agit de recréer. Le plan de 1991 précise : « Notre but est de restaurer le paysage originel, avec ses canaux, ses îles et sa verdure, et les expériences du passé, telles que les fêtes locales et les jeux dans les canaux ». Et celui de 1994 veut reconvertir le site d'un haut-fourneau en « village d'artisanat et de jardinage ».

37. Kasagi Hiroo, "Initiatives by pollution patients to renew their communities", ibid., p. 28. Idem pour toutes les citations suivantes (pp. 25-26). 
Le thème de la symbiose figure aussi en bonne place. Plantes et animaux sont classés comme victimes au même titre que les hommes. Et les entreprises pollueuses sont invitées de grand cœur à réintégrer la communauté : «Il est vital de retisser les liens entre les entreprises et les citoyens (...) sous la forme d'une relation de coopération et de partenariat. Tous membres d'une même localité, ils en feront une véritable communauté pleine de vie, où il leur sera facile de vivre ensemble »38. Un discours qui plaît d'autant plus aux firmes qu'elles possèdent de larges terrains inutilisés dans la zone polluée, dont la restauration accroîtra la valeur... Les militants communistes ne s'y trompent pas, qui continuent à dénoncer l'administration et les entreprises, à prôner le retour des pouvoirs locaux de gauche comme solution ultime aux problèmes d'environnement et à lier « l'éradication de la pollution et la promotion de la paix », dans la tradition rhétorique de la gauche japonaise 39.

Malgré ces divergences, les deux mouvances sont parvenues à collaborer pour faire entrer le mouvement dans une nouvelle étape, qui constitue un approfondissement majeur. Nishiyodogawa est passé de la lutte défensive pour les indemnisation à la prise en charge "directe et démocratique" du problème de la pollution par la communauté tout entière, qui se reconstruira à travers le processus. Nous retrouvons ici clairement les idéaux des Clubs et de Green Coop.

Tous les mouvements de lutte locaux ne connaissent pas la même réussite. Mais leurs échecs ne sont pas moins instructifs, à l'exemple de celui du mouvement anti-nucléaire de Hokkaïdo.

\section{LES LECONS D'UN ÉCHEC : LA LUTTE ANTI-NUCLÉAIRE À HOKKAÏDO 40}

\subsection{Un mouvement trahi par le mura et par la gauche}

Le mouvement contre la centrale nucléaire de Tomari, à Hokkaïdo, concerne quatre villages de pêcheurs et paysans. À ses débuts (1970), il rassemble des activistes de gauche, notamment des enseignants affiliés au syndicat socialiste

\section{Ibid.}

39. Blue Sky for Children, rapport de la Japan Air Pollution Association, Tokyo, 1996.

40. Ce passage se fonde sur l'interview de M. Someya Kazuhiko, responsable de la Société de réflexion sur le problème nucléaire d'Iwanai (Iwanai genpatsu mondai kenkyûkai), le 14 octobre 1996 et sur l'ouvrage de KASHIMA Yotarô, Warera sabishiki genjûmin ("Nous, pauvres autochtones" ou "Nous, pauvres citoyens nucléaires" : il y a un jeu de mot), Sapporo : Image Office, 1993. 
Sôhyô ${ }^{41}$, et les coopératives de pêcheurs et d'agriculteurs qui craignent la mévente de leurs produits. Le mouvement utilise les structures des mura et surtout leurs organisations de jeunes (seinenbu). Pendant plus de dix ans, celles-ci mènent l'action sur le mode ancestral de la supplique et de la discussion avec les élus. Au long de ces palabres, la Cie d'Electricité de Hokkaïdo (Hokuden) porte ses offres de dédommagement à 8,34 milliards de yen. Les coopératives, qui ne cherchaient sans doute pas autre chose, se laissent circonvenir. Acculé, le mouvement recourt tardivement à la confrontation. Mais une pétition pour obtenir la démission du maire (pro-nucléaire) du village de Kyowa échoue. C'est la fin de la résistance des mura. Les travaux commencent en 1984.

Le mouvement change de stratégie. Hokkaïdo est un bastion du Parti socialiste et de son syndicat, Sôhyô. Pour 1987, ils sc préparcnt à rcconquérir lc poste de gouverneur, perdu en 1979. Leur candidat, Yokomichi Takahiro, promet de faire cesser les travaux. Sôhyô crée un comité anti-nucléaire, Zendô rôkyô (Association des travailleurs de Hokkaïdo). Les citoyens sont mobilisés à travers un shimin undô de type classique : Han genpatsu zendô jûmin kaigi (Ligue des citoyens de Hokkaïdo contre le nucléaire). Mais sitôt élu, Yokomichi oublie ses promesse. La Ligue tente alors la démocratie directe. Elle lance une pétition pour demander au conseil général d'organiser un référendum (1988). Elle recueille 900000 signatures ; 250000 suffisaient. Au conseil général, entre 54 conservateurs qui rejettent la pétition et 52 élus de gauche qui l'appuient, les voix décisives sont celles des trois élus du Minsei Club. Ils sont liés au syndicat modéré Dômei, mais très proches du gouverneur. Deux votent contre la pétition. Ce qui aurait été le premier référendum régional jamais organisé au Japon n'aura pas lieu.

La Ligue se décide alors, bien tard, à recourir à la justice (1989). 50000 personnes lancent Tomari genpatsu unten sashidomei soshô ("La plainte en justice pour stopper la centrale nucléaire de Tomari"). Chacune verse 1000 yen pour financer la procédure intentée par 150 "plaignants délégués". Cinq "permanents" assistent à toutes les audiences, sous le contrôle d'un comité de 31 membres. Mais, aujourd'hui, deux tranches de la centrale fonctionnent et une troisième est en voie d'achèvement. Le successeur de Yokomichi, élu après avoir fait campagne contre, a lui aussi retourné sa veste. Il n'y a plus guère que les quatre élues du NET au conseil municipal de Sapporo pour mener encore le combat dans les assemblées locales. La Ligue ne compte plus qu'une dizaine d'activistes permanents, et "La plainte..." ne publie même pas de newsletter, faute de moyens.

41. Notre informateur est d'ailleurs permanent de la Fédération des enseignants de Sôhyô. 


\subsection{Les leçons d'un échec}

Le cas de Tomari révèle quelques uns des problèmes majeurs auquel se trouve confronté le mouvement de la "reconstruction de la démocratie par le bas" au Japon :

a) L'utilisation des structures des mura peut être efficace comme mode d'organisation, mais leur mobilisation ès-qualité avorte dès qu' elles atteignent leurs objectifs immédiats (indemnisation). Même si leur fonctionnement et leur imaginaire collectif sont porteurs de démocratie directe, elles sont peu capables de dépasser l'intérêt de leur localité. Les matrices communautaires traditionnelles doivent être investies d'un contenu idéologique nouveau. A défaut, le mouvement achoppe sur l'esprit de clocher et le clientélisme conservateur reprend le dessus.

b) Les partis de gauche, intégrés dans le jeu de l'Etat "néo-corporatiste", ne constituent pas des alliés fiables. Yokomichi a trahi les antinucléaires. Si Sôhyô les a soutenus, c'est parce que sa très puissante fédération des enseignants la tirait et qu'elle n'avait rien à perdre à Hokuden, avec 150 adhérents contre 3000 au syndicat-maison. Et le Parti socialiste a montré qu'il n'attachait aucune importance à ses liens avec la mouvance alternative quand il a abandonné son opposition de principe au nucléaire civil pour gouverner avec le PLD en 1994. A Nishiyodogawa, l'existence d'un relais politique local et le lien avec le Parti communiste ont beaucoup aidé au succès initial du mouvement. Inversement, l'absence de relais politique fiable a sapé le mouvement de Hokkaïdo, sans qu'il soit capable d'imaginer une stratégie pour s'en passer, ni assez audacieux pour affronter le gouvernement régional, en sus des mura, de Tokyo et de Hokuden...

c) Les alternatifs n'ont pas confiance dans l'institution judiciaire et sont assez inhabiles à l'utiliser. Ils ont attendu dix-neuf ans avant de s'adresser à elle. Même alors, leur stratégie vise à obtenir des retombées médiatiques plutôt qu'une décision favorable. Ils privilégient les actions spectaculaires, comme faire avaler de force au juge du lait des pâturages de Tomari, pendant que les sympathisants manifestent violemment devant le tribunal (ce qui a amené le magistrat à suspendre les audiences pendant deux ans). Il est vrai que les bases juridiques sont fragiles. Au civil, les plaignants doivent prouver qu'ils ont subi un dommage personnel ; mais aucune atteinte à la santé n'est détectable dans l'immédiat et les pertes économiques ont été indemnisées par Hokuden. Un recours administratif contentieux ne coûte que 10000 yen de droit de timbre, mais il faudrait prouver que la Loi sur les centrales nucléaire viole la Constitution - un argument qui n'a aucune chance en Cour suprême ; ou que la procédure d'autorisation a été entachée d'irrégularités - ce que les plaignants ne peuvent pas prouver faute d'avoir accès aux dossiers administratifs; ou que les études d'impact sur l'environnement n'ont pas été menées correctement. Mais sur ce point, il n'existe que des "directives" (gyôsei) locales éparses - Hokkaïdo en a une - dont la force légale fait l'objet d'une jurisprudence confuse. 
d) Le mouvement est peu ouvert sur l'extérieur. Hokkaïdo compte plus de 600 groupes écologiques, comme ceux qui s'opposent aux terrains de golf, mais les antinucléaires n'ont pas réussi à les fédérer. La Ligue coopère avec deux mouvements du nord de Honshu, à Wakanai (stockage de déchets) et Rokkasho, site candidat pour un accélérateur de particules. Mais elle n'a envoyé que 25 militants aux manifestations de 1996 à Rokkasho. Au plan international, Greenpeace n'a été contacté qu'en 1995 et la relation reste faible : M. Kashiwa la définit comme « cooperating, but not working together ». La Ligue ne veut ni aide financière, ni assistance organisationnelle. Des missions ont été envoyées depuis 1995 à La Hague, en Corée, à Taïwan et dans le Pacifique Sud : dans ce cas, c'est « gathering, not working together». L'action a été plus résolue autour de Tchernobyl, où $\mathbf{M}$. Kashiwa s'est rendu (collecte de fonds, accueil d'enfants contaminés). Mais la résolution pour passer à 1'international semble manquer. «C'est trop pour nous », assure M. Kashiwa. Après vingt-cinq ans, l'activisme semble être devenu un mode de vie qui se suffit à lui-même et dont les militants, marqués par les déceptions, craignent qu'il soit bouleversé.

e) Les antinucléaires sont très mal organisés au plan national. Il sont divisés entre les pragmatistes, qui veulent geler le programme nucléaire en combattant les projets d'extension, et les radicaux qui veulent dénucléariser. En l'absence d'exécutif permanent, la coordination est plus ou moins assurée par deux universitaires, Takagi Junzaburo (Université nationale de Tokyo) et Fujita Ryuko (Université Keio), dont les bases de données contiennent les coordonnées des antinucléaires du Japon. Les Clubs et le NET jouent aussi un rôle. Kanagawa NET, auquel Fujita est très lié, a créé une "hotline" antinucléaire. A Hokkaïdo, le Club a créé le mouvement "Sayonara genshiryoku hatsuden" ("Bye-bye, centrales nucléaires !"). Il existe aussi un réseau d'aide aux ouvriers victimes de contaminations (Hibakusha rodosha kyoen nettowôku), que les compagnies cherchent à dissimuler. Pour l'action politique, les antinucléaires sont aussi divisés que les autres alternatifs. Aux législatives de 1996, Takagi s'est violemment opposé à ce que Kanagawa NET soutienne le Minshutô. A Hokkaïdo, le mouvement a refusé d'accorder le moindre suisen et n'a organisé aucune action électorale.

f) Le mouvement a mal résisté à ses échecs. Il n'a pas su rebondir après sa très courte défaite de 1989 en capitalisant sur l'élan de la pétition. Cela tient sans doute à son caractère défensif. La Ligue ne lutte pas pour la mise en place de formes alternatives de vie quotidienne. Son agenda est imposé par les élites qui poursuivent un objectif déterminé. Elle ne définit pas elle-même son champ d'action. La "réinvention de la démocratie" se déploie mieux de son propre mouvement, et vers l'avenir.

Mais, par-delà ses faiblesses, le mouvement a aussi montré que les contestataires disposent d'une gamme de stratégies très étendue. Surtout, les militants de Tomari ont ouvert la voie aux deux référendums locaux victorieux de 1996 (contre la centrale nucléaire de Maki en août et contre les bases américaines 
d'Okinawa en septembre). Deux initiatives qui remettent aujourd'hui en cause tout l'équilibre des pouvoirs au Japon.

\section{ET SI GRAMSCI AVAIT RAISON ?}

\subsection{Essouflement et rebond des coopératives alternatives}

Depuis quelques années, les coopératives alternatives marquent le pas. Les Clubs n'ont créé aucune branche régionale depuis 1990 et leurs effectifs commencent à baisser. A Kanagawa, ils ont perdu 1968 membres entre 1994 et 1995. A Hokkaïdo, après une forte expansion de 5000 membres en 1982 à 14000 en 1992, le Club est retombé à 12920 en 1995 42. De l'avis même de ses responsables, le mouvement traverse une crise de maturité. Il s'est banalisé. Il y a dix ans, les femmes y adhéraient à une cause et gardaient leur affiliation plus ou moins secrète. Aujourd'hui, les Japonaises sont libres de s'engager au gré de leurs aspirations dans les mouvements les plus divers. Les coopératives autogérées rebutent les jeunes par ce qu'elles exigent d'investissement personnel et de discipline. Leurs adhérentes typiques sont des ménagères aisées de plus de 45 ans. Au NET, les trois quarts des élues sont épouses de cadres, de membres des professions libérales ou de petits patrons ; plus de la moitié ont un revenu annuel supérieur à huit millions de yen (350 000 F) 43 . Ce n'est pas un mince paradoxe que de fonder un mouvement contestataire sur une base composée surtout de femmes mûres de la moyenne bourgeoisie... De manière générale, c'est toute la première génération d'alternatifs - surtout des anciens de ' 68 nourris d'idéologie, très militants et très organisés - qui se trouve décalée par rapport aux aspirations de plus en plus hédonistes de la société 44 . Et avec l'élévation du niveau de vie et l'essor de la grande distribution depuis la déréglementation, la vente de produits en gros, fussent-ils biologiques, n'est pas une activité d'avenir.

Face à cette situation, on a vu que les Clubs ont une stratégie d'approfondissement, qui passe par la diversification des activités et la multiplication des workers' collectives. Mais beaucoup critiquent aussi la duplication systématique des schémas d'organisation de la structure-mère de Tokyo-Kanagawa. Hokkaïdo veut assouplir les conditions d'entrée et l'organisation des han, au risque d'y perdre un peu de l'âme des pionniers. En 1994, le Club de Tokyo s'est restructuré en quatre entités de taille plus réduite. Kanagawa a même dérogé aux sacro-saints principes en ouvrant 16 Community Clubs qui ressemblent fort à des boutiques, même si les membres y doivent trois périodes de service par an.

42. Chuppu, $\mathrm{n}^{\circ} 160$, mai 1996, p. 6.

43. Lam Peng-Er, op. cit., pp. 111-112.

44. Sur ce point, voir Jean-Marie BouISSOU et Éric SEIZELET, op. cit. 
Au plan politique, l'action des alternatifs est contrariée par leur aversion pour les partis et la volonté d'autonomie farouche de chaque NET régional. Pourtant, ils sont peut-être en train de gagner le pari gramscien. Leur esprit gagne les mouvements de lutte locaux, comme à Nishiyodogawa. Mais leur thématique contamine aussi de plus en plus le discours politique au niveau national et des personnages, directement issus de la mouvance alternative, jouent aujourd'hui un rôle de premier plan à la Diète.

\subsection{La contamination au sommet : de Doi à Kan}

Déjà aux élections sénatoriales de 1989 , le Parti socialiste avait fait élire une dizaine d'activistes, en majorité des femmes, issus des mouvements de lutte locaux et des organisations de consommateurs. Une poignée entra aussi à la Chambre, sous l'étiquette socialiste, aux législatives de 1990. A cette occasion, le NET accorda pour la première fois sa recommandation à des candidats. A Kanagawa-4, M. Ikeda Motohisa fut élu comme socialiste grâce à la mobilisation des Clubs, malgré la mauvaise volonté de l'appareil local du parti. A Kanagawa-2, Mme Yokohama Sumiko, indépendante, recueillit 83354 voix, mais fut battue.

Ce rapprochement avec le Japon contestataire et associatif devait tout à Mme Doi Takako. Cette universitaire, sans lien avec aucun lobby, était devenue présidente du parti après que sa débâcle électorale de 1986 eût discrédité les dinosaures syndicalistes qui en confisquaient la direction. Sa stratégie donna aux socialistes leurs deux plus beaux succès électoraux de l'après-guerre, en devançant le PLD aux sénatoriales et en obtenant $24,4 \%$ des suffrages $(+7,2)$ et 136 sièges $(+51)$ aux législatives. Mais les gérontes syndicaux vivaient très mal cette intrusion d'éléments incontrôlés. Dès 1991, ils évincèrent Mme Doi et tournèrent le dos aux alternatifs en "droitisant" le parti, jusqu'à accepter le nucléaire civil. Ce faisant, ils causèrent sa perte : $15,4 \%$ des suffrages et 75 sièges en $1993,6,4 \%$ et 15 sièges en 1996.

Cet effondrement prouva, a posteriori, que les réseaux du Japon contestataire et alternatif constituaient l'une des rares forces organisées capables de soutenir des candidats non conservateurs face aux kôenkai du PLD. Traditionnellement, ce rôle revenait aux syndicats ; rares étaient les députés, tel Kan Naoto à Tokyo, qui utilisaient déjà les réseaux alternatifs comme machine électorale 45. Aujourd'hui, cependant, les syndicats sont en pleine crise, comme toutes les structures de "l'État néo-corporatiste". Reste le Japon associatif. Sur la base de 1,7 vote par membre (celle qui sert à mesurer la capacité mobilisatrice des kôenkai), les seules coopératives alternatives commande-

45. Voir Jean-Marie Bouissou et Kan Naoto, "Un mutant au Parlement", Pouvoirs, $n^{\circ} 71$ (Le Nouveau Japon), pp. 67-72. 
raient environ un million de voix, dont 230000 à Fukuoka, 120000 à Kanagawa et 95000 à Tokyo. Cela reste marginal : $1 \%$ du corps électoral à Tokyo, $1,8 \%$ à Kanagawa, mais tout de même $6 \%$ à Fukuoka. Mais, avec l'explosion de l'abstention, cela a peut-être pesé $10 \%$ des votants à Fukuoka aux législatives de $1996,3,3 \%$ à Kanagawa et $1,8 \%$ à Tokyo. Assez pour faire la différence dans un nombre non négligeable de circonscriptions. Et, avec une loi qui limite drastiquement toutes les formes de campagne, au moment des élections, le moindre groupe militant organisé a une importance considérable, que la versatilité des électeurs accroît encore...

Au-delà des élections, l'importance des alternatifs se mesure désormais au fait que les thèmes, qui sont depuis toujours au centre de leur discours, font florès dans les médias, et mouche auprès des électeurs flottants : retour du pouvoir vers le local, libération de l'initiative individuelle et de l'action citoyenne, fin des rigidités hiérarchiques, changement qualitatif de la croissance, "amateurisme politique aux mains propres". Le Japon associatif, où les alternatifs jouent un rôle majeur, est ainsi devenu un élément essentiel de la recomposition du système des partis. Les élites nationales sont obligées de le prendre en compte, pour le mobiliser ou pour s'en défendre. On peut donc dire qu'il occupe à présent sur la scène politique une position incontournable, sinon centrale.

\subsection{Naissance du Minshutô (20 septembre 1996)}

C'est dans ce contexte que nait le Minshutô (Parti démocrate), en septembre 1996. Ses deux fondateurs sont Hatoyama Yukio et Kan Naoto. Le premier, ancien du PLD, est l'héritier d'une vieille dynastie et d'une formidable fortune. Kan vient de la petite classe moyenne et de la mouvance alternative. Ancien de ' 68 , député de Tokyo depuis 1980 sous l'étiquette d'un groupuscule socialisant (le Shaminren), il est lié de longue date aux Clubs, qui hui fournissent l'essentiel de son soutien électoral organisé. Sa nomination à la Santé, dans le deuxième cabinet socialiste-PLD (1995-1996), s'explique d'abord par là, puisque ce Ministère contrôle les coopératives de consommation. Kan y gagne une formidable popularité avec l'affaire du sang contaminé. Les hémophiles victimes du virus HIV luttaient depuis le début des années 1990 pour établir les responsabilités dans leur drame. Le Ministère prétendait n'avoir aucun dossier sur l'affaire. Sitôt nommé, Kan obligea ses fonctionnaires à lui remettre les documents qui dévoilaient leur collusion avec les firmes pharmaceutiques. Les responsables du lobby médico-bureaucratique sont aujourd'hui inculpés, alors que le porte-parole des hémophiles a été élu député du Minshutô en 1996. Kan est devenu l'homme politique le plus populaire du pays et le seul qui puisse mobiliser le Japon contestataire et associatif.

Hatoyama (50 ans) et Kan (51 ans) sont de la même génération. Ils disposent tous les deux de ressources politiques propres (un formidable héritage pour l'un, un ancrage électoral personnel pour l'autre) qui les rendent indépendants des appareils partisans. Ils partagent la même aversion pour les gérontes du 
PLD, les syndicats et les bureaucrates qui gèrent la "société verticale". Tous deux ont évité de s'user dans les manœuvres confuses de la recomposition politique entre 1993 et 1996 . Ils ne sortent du bois qu'à la veille de la dissolution. Le Parti socialiste et le Parti pionnier (centre-gauche), qui ont vendu leur âme pour gouverner avec le PLD depuis 1994, sont à l'agonie : popularité quasi nulle, caisses vides... Leurs sortants sont prêts à saisir n'importe quelle bouée. Kan amène une popularité inégalée, Hatoyama de l'argent et des réseaux : 52 députés adhèrent en hâte au Minshutô.

Le nouveau parti se comporte honorablement le 20 octobre. Il maintient ses positions (52 sièges) et obtient $16 \%$ des voix à la proportionnelle. Avec un seul mois pour s'organiser, il n'a pas trouvé de candidats pour 157 des 300 circonscriptions locales. Parmi ses nouveaux élus, on retrouve Ikeda Motohisa, le socialiste/alternatif de Kanagawa, battu en 1993 par les bons soins de son propre parti et réélu dans la 6ème circonscription avec l'appui du NET.

\subsection{Le discours du Minshutô, ou le triomphe de la thématique alternative}

Le préambule à la plate-forme politique du Minshutô 46 est un véritable répertoire des images et des concepts de la mouvance alternative japonaise.

Le refus de la "société verticale" : «Depuis cent ans, nous avons connu un système centralisé et vertical, où la démocratie s'est développée d'en haut, sous la protection de la bureaucratie (...) Il faut bâtir maintenant une société centrée sur les citoyens, multipolarisée, horizontale et coopérative ».

L'affirmation d'une volonté de changer de monde, en refusant tous les systèmes existants : «Nous avons décidé d'agir pour réformer complètement la structure sociale de ce pays (...) Le libéralisme capitaliste et l'égalitarisme socialiste - les deux idées qui ont fait bouger le monde - rivalisaient en apparence. Mais leur point commun est de traiter les hommes comme une masse sans visage, tout comme le système japonais de "capitalisme égalitariste" sous contrôle bureaucratique ».

Le refus des partis au profit des réseaux interactifs de la "cyber-démocratie" : «Nous développerons une structure politique de réseaux horizontaux. Dans cette structure, si quelqu'un découvre un problème et propose une solution, il trouvera des gens qui sympathiseront, et des "carrefours" se créeront. Une fois le problème résolu, la structure reviendra à son état d'origine. Cela ressemblera au système immunologique du corps humain. (...) Pour gérer ce type de réseau politique, nous utiliserons massivement les moyens de communication électroniques. Nous serons la première génération de cyber-citoyens globaux ».

46. Traduit à partir du texte miméo, dont je remercie Banri Kaieda, député de Tokyo, de me l'avoir fourni avant publication. 
L'appel à la participation active de tous : «Il faut propager un style d'action et une conscience qui soient ceux de citoyens globaux du monde, qui s'engageront dans la décision politique au niveau local, au niveau national et au niveau mondial, selon leur sensibilité et leurs intérêts (...) des citoyens agents de la politique, qui s'informeront et penseront par eux-mêmes, et dépenseront leur argent et leur énergie pour réaliser une vie meilleure ».

La société de petites communautés autonomes : «Un petit gouvernement, un petit parlement, des pouvoirs locaux puissants et efficaces, (...) le transfert de la souveraineté de l'Etat vers les provinces ».

Une autre forme de croissance, à échelle humaine et coopérative : "Nous allons voir reculer la production industrielle massive, la consommation massive et la pollution massive (...) Nous verrons se développer de nouveaux domaines d'innovation technologique, des PME créatives et riches de savoir, des agriculteurs autonomes et des secteurs actifs d'économie citoyenne (ONG et coopératives). Ils créeront une économie de marché, de symbiose et de circulation des ressources, assurant une croissance régulière ».

L'idéal d' une société sans contrainte, fonctionnant “à la symbiose”, version écologiste : «L'esprit que nous voulons pour base de la société à venir, c'est la fraternité (...) Chacun de nous est un être irremplaçable (...) qui a le droit de décider lui-même de son sort et le devoir d'assumer son choix. C'est cela, l'autonomie de l'individu. Nous devons viser à la symbiose avec les autres, en respectant l'autonomie et la différence de chacun, et vivre en empathie (...) Autonomie et symbiose valent pour les relations au sein de la société japonaise, mais aussi entre le Japon et le monde, et même entre les hommes et la nature. La conception chrétienne de la fraternité (...) apprend à aimer son prochain (...) Mais la sagesse orientale enseigne que les hommes font partie de la nature. Un arbre ou un animal sont des êtres aussi irremplaçables que nous. Il est donc possible de réaliser un échange profond entre la nature et les hommes. C'est l'esprit de fraternité propre à l'Orient (...) qui se fonde sur l'empathie avec la nature et le fait de chérir la vie ».

Cette rhétorique peut prêter à sourire. Mais l'état d'esprit qu'elle reflète sous-tend des évolutions en profondeur qui font trembler les élites conservatrices.

* Le rééquilibrage du pouvoir au profit de communautés locales de plus en plus combatives. La décentralisation a été l'un des thèmes qui ont porté les partis "néo-conservateurs" en 1993. Après son retour aux affaires, le PLD a fait voter une timide loi de décentralisation (1995). Mais les pouvoirs locaux ne s'en contenteront pas. Une étape décisive a été l'organisation de deux référendums locaux sur des sujets cruciaux. En août 1996, les habitants de la petite ville de Maki ont rejeté l'implantation d'une centrale nucléaire. En septembre, à l'appel de leur gouveneur, ceux d'Okinawa se sont prononcé contre les bases militaires américaines dans leur île. Bien que dépourvues de force contraignante, ces consultations marquent une étape essentielle dans le rééquilibrage du pouvoir en faveur des chiiki chers aux Clubs. En mars 1997, la puissante 
Cie d'électricité du Kyushu a renoncé à construire une nouvelle centrale dès que les opposants ont parlé de référendum. Le mouvement de Hokkaïdo envisage de retenter sa chance avec une nouvelle pétition. C'est tout le programme nucléaire civil, déjà très en retard sur ses objectifs, qui est menacé de paralysie.

Le débat sur la réforme de la Constitution, qui a eu lieu à l'occasion de son 50 e anniversaire, a montré que les citoyens souhaitaient surtout pouvoir exprimer directement leur volonté, par l'élection du premier ministre au suffrage universel et l'extension de la procédure de référendum 47 . Si le PLD y est très hostile, il se trouve pourtant quelques uns de ses dirigeants, comme l'ancien premier ministre Nakasone, pour soutenir la première demande. Preuve que les temps changent... Pour apprécier la portée de ce rééquilibrage, il faut se souvenir que l'interdiction absolue du cumul des mandats fait des élites politiques locales et nationales deux mondes séparés. Il faut aussi se rappeler que, depuis trente ans, nombre de changements majeurs ont été initiés par les pouvoirs locaux, qui ont obligé le gouvernement PLD à les suivre. Le modèle de la politique de soutien aux PME a été donné par le gouverneur communiste de Kyoto ; la gratuité des soins pour les personnes âgées, par le gouverneur socialiste de Tokyo; les mesures anti-pollution, par la municipalité d'Osaka ; et pour l'amélioration du statut des résidents étrangers, le maire de Kawasaki n'a pas craint de désobéir ouvertement au gouvernement en 1985. Le glissement du pouvoir vers les communautés locales ne pourra donc manquer de modifier profondément les politiques au niveau national.

* Le rééquilibrage des pouvoirs va de pair avec une combativité croissante des citoyens contre l'administration. L'obstination des hémophiles face au Ministère de la santé a valeur de symbole. La société civile reprend à sa manière le thème de la "réforme administrative" utilisé dans les jeux de pouvoir entre élites, en exigeant "la transparence de l'information" (jôhô kôkai). De nombreux procès sont en cours pour obtenir l'accès aux documents les plus divers, depuis les livrets scolaires jusqu'aux frais de représentation des fonctionnaires et des élus. Les contribuables ont déjà obtenu la démission du gouverneur d'Akita et le remboursement de dizaines de millions de yen par les ken de Hokkaïdo et de Tokyo. La justice leur a même reconnu le droit de connaître le détail des dîners payés avec leurs impôts. Et les antinucléaires de Hokkaïdo ont relancé leur combat judiciaire en entamant une deuxième procédure pour que Hokuden rende public le manuel de sécurité de ses installations, au nom du jôhô kôkai. Le PLD, prisonnier de la rhétorique anti-bureaucratique obligée, ne peut plus éluder la mise en chantier d'une loi sur ce sujet. D'autant plus que les pouvoirs locaux, comme à Fukuoka, ont déjà pris les devants en édictant leurs propres règlements.

47. Philippe PoNs, “Le Japon, l’épée au fourreau”, Le Monde du 7 juin 1997. 
* La situation politique offre une opportunité exceptionnelle pour les revendications citoyennes. Le PLD est minoritaire à la Diète. Il a besoin de l'appui au moins tacite d'un des deux partis d'opposition : le Shinshintô à droite, le Minshutô à gauche. Or ceux-ci ne peuvent pas espérer parvenir un jour au pouvoir sans reprendre les demandes de la société civile. Pour les faire avancer, le Minshutô pratique le marchandage au coup par coup ; ainsi, il a appuyé le PLD dans l'affaire des bases d'Okinawa contre la promesse d'étudier la création d'une agence indépendante pour contrôler l'administration, sur le modèle du Board of Audit des États-Unis. Le Shinshintô ne peut pas paraître en retrait. Tous les partis doivent donc surenchérir sur les thèmes qui sont au coeur de l'agenda des alternatifs.

On peut évidemment se demander si cette surenchère ne va pas servir seulement à établir une nouvelle donne entre les élites. L'avènement de "l'ère des régions" (chihô no jidai) sera-t-il autre chose qu'une revanche des élites locales sur l'appareil du PLD et sur les dynasties politiques qui accaparent les sièges parlementaires ? L'appel au changement est-il autre chose qu'une stratégie discursive utilisée par la génération d'après-guerre, qui entre dans la cinquantaine et aspire à se saisir enfin des rênes, à Tokyo comme au niveau local 48 ? Une fois que les pouvoirs seront redistribués, qui parlera encore de "réseaux politiques d'un type nouveau" ou de "symbiose entre l'homme et la nature" ? Bref, le mouvement alternatif va-t-il être récupéré comme jadis les mouvements d'habitants ou de consommateurs 49 ?

La réponse dépendra de sa capacité à surmonter sa difficulté à se coordonner au niveau national, l'étroitesse de sa base sociologique et de son agenda et l'ambiguïté de ses relations avec les pouvoirs, symbolisée par le discours sur la "symbiose". Ce qui ramène à six facteurs :

a) La vivacité et la durée de l'affrontement entre élites locales et nationales. C'est lui qui pousse gouverneurs et maires à organiser des référendums, à prôner la participation des citoyens et à reprendre aux alternatifs leurs moyens d'action pour se légitimer. Plus la compétition sera acharnée, mieux les alternatifs pourront en profiter.

b) La capacité des alternatifs d'établir un dialogue avec les syndicats. Ceuxci constituent toujours une base essentielle de soutien organisé aux forces antiPLD. Ils ont désespérément besoin d'un souffle nouveau. Même si le contentieux est lourd entre eux et la mouvance alternative, ils ne peuvent pas ignorer où sont aujourd'hui les forces vives du militantisme.

48. Pour l'utilisation de la thématique alternative dans les luttes entre élites locales, voir les cas de Hakata-bu benkyôkai et de la commune de Niseko, dans Jean-Marie Bouissou, "Un autre Japon", op. cit.

49. Pour les jeux de récupération/contre-récupération entre alternatifs et autorités, voir les cas du "mouvement des volontaires" et du mura okoshi undô ; ibid. 
c) Leur capacité de mobiliser la jeunesse, qui oscille entre un conservatisme et un cynisme, également confortables, et une révolte molle et ludique symbolisée par les "néo-nouvelles" religions. Mais la sociologie de la mouvance alternative et de ses dirigeants laisse penser que ce sera difficile.

d) La profondeur de la "contamination" du niveau national par la thématique alternative. Elle est difficile à apprécier. La présence de Yokomichi dans les rangs du Minshutô n'est pas du meilleur augure. Mais à force de s'agenouiller devant les icônes de l'imagerie alternative, certains leaders politiques finiront peut-être par devenir croyants...

e) La capacité des alternatifs d'utiliser les formes modernes de la corrmunication pour surmonter leur incapacité chronique à se coordonner.

f) Enfin, et peut-être surtout, leur capacité de sortir du cadre national, pour contribuer à l'invention de la future idéologie qui, tôt ou tard, s'affirmera contre l'ultra-libéralisme et les sacro-saints "marchés". Au même titre que les Grünen ou les Verts, les alternatifs japonais sont porteurs d'une expérience originale, à travers laquelle leur pays pourrait participer, pour la première fois de son histoire, à la recomposition de la scène idéologique mondiale. 